\title{
The Design and Implementation of Hybrid Engine used in English interaction learning system
}

\author{
Yong $\mathrm{Fu}$ \\ Zhengzhou University of industrial technology, Henan, China
}

\begin{abstract}
With the continuous improvement and development of Hybrid recognition technology, the numerous special purpose chips for Hybrid recognition have been developed, thus, the practical products of Hybrid recognition have been gradually appeared in the market. This paper will take the foundation of Hybrid coding in English embedded Hybrid system as the breakthrough point, by means of the interpretation of Speex, it discusses the design of the Hybrid engine as well as the route of implementation so as to reduce tedious work in voice testing. Combined with the analysis of the basic structure of Hybrid recognition in the embedded system, it discusses the architecture of the speaker independent English Hybrid recognition learning system.
\end{abstract}

Keywords: Hybrid coding; Speex; Hybrid engine; Embedded Hybrid learning system.

\section{Introduction}

Hybrid coding is the basic technology of digital Hybrid transmission and storage, by means of the compressed digital, it can represent the Hybrid signals and make the expression of these signals with the minimum number of the required bits. Compared with the stimulated voice, digital voice transmission and storage system of using Hybrid coding technology, has the advantages of high reliability, strong anti-interference ability, easy to be quickly exchanged, easy for the realization of confidentiality, multiplexing, packaging as well as the advantage of low price, etc. The compressed voice is used for transmission, which can reduce the required bandwidth of each route, thus it can transmit mote voice transmission in the same bandwidth; it can be used for storage, which can save space and improve the storage of Hybrid length as well as reduce cost. With the development of computer technology, signal processing as well as the development of pattern recognition technology, Hybrid recognition technology has been improved gradually, the fields of application have been more and more extensive, at the same time, a lot of Hybrid recognition products have been appeared. Hybrid recognition products are widely used in voice dialing system, English and Chinese translation system, intelligent toys controlling, intelligent home controlling system, smart phones, stock trading system, banking service system, medical intelligence service, the intelligent automobile navigation, industrial control and some other fields.

\section{The Interpretation of Speex}

Speex is a multi-mode, multi-rate, Hybrid code, based on CELP algorithm, it can provide narrow band, wide band and ultra-wide band three Hybrid codec modes, which are respectively corresponding to the Hybrid signals with the bandwidth of $4 \mathrm{kHz}$ (sampling rate is 8000 ), $8 \mathrm{kHz}$ (sampling rate is 16000), 16kHz (sampling rate is 32000) [1]. Among them, the narrow band Hybrid coding only adopts narrow band sub-pattern coding; wide band Hybrid can be divided into two sub bands, wide band Hybrid adopts broadband sub-pattern coding, low band voice adopts narrow band sub-pattern coding; ultra-wide band will repeat decomposition two times which adopts the wide band sub-pattern coding twice and narrow band sub-pattern coding once. Thus, we can see, throughout the algorithm of Speex, it is composed by two types of sub-pattern encoding: narrow band sub-pattern and wide band sub-pattern. 


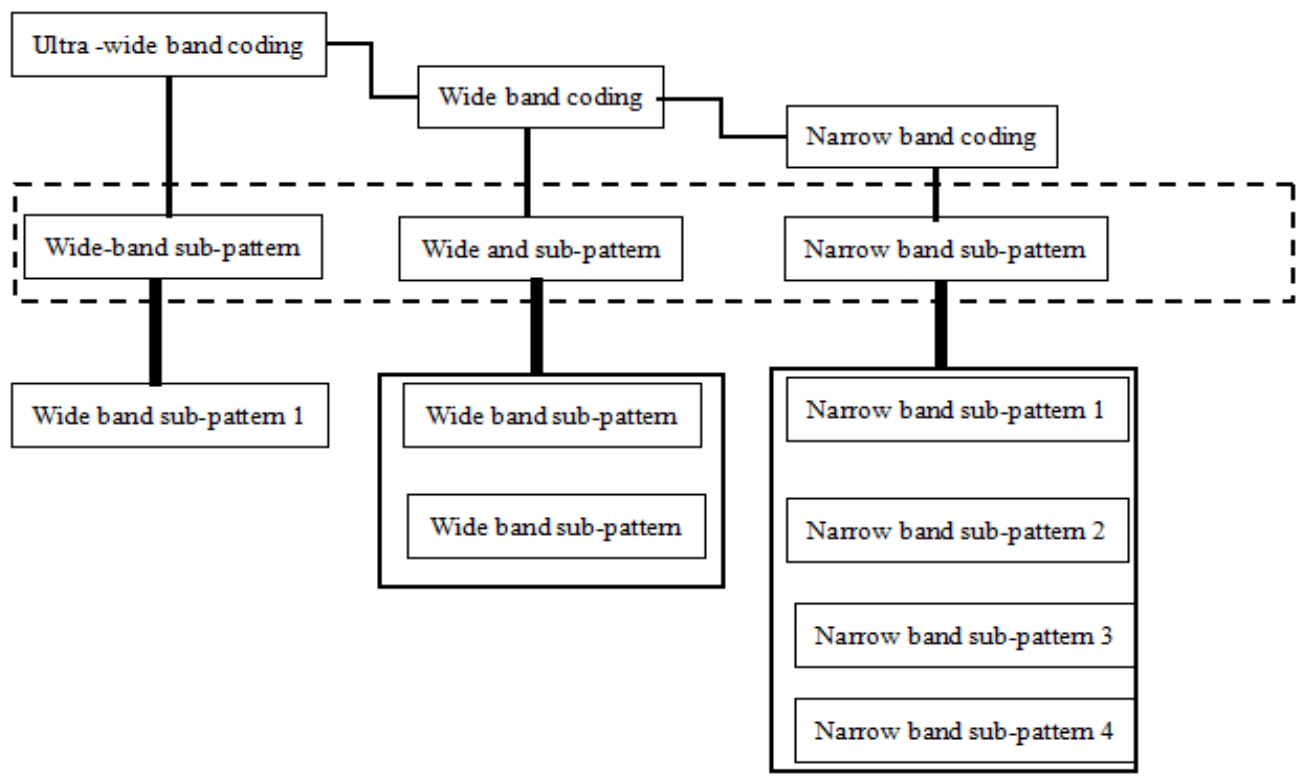

Fig 1. Decomposition of coding mode

\section{The Structure of the Functional Module of the English embedded interaction learning System}

In this paper, VOIP system adopts Speex coding. The whole system consists of two parts, the server side and client side. The server of the database saves the data of all registered users. Each user must firstly log on the server used by the client side, and access to the list if online friends, then it can make a voice call to the online friends. The call between the client sides adopts the mode of point to point [2]. It can avoid the excessive delay of voice packet caused by the transmission of the server side. English embedded interaction learning system refers to the application of using various advanced microprocessor with the realization of Hybrid recognition technology in board-level or chip-level with software or hardware. English embedded interaction learning system is required to achieve the optimization of algorithm under the premise of ensuring the recognition effect as much as possible, so as to adapt to the characteristics of the embedded platform with less storage resources and real-time. The large vocabulary continuous English embedded interaction learning system with high performance in the advanced level of laboratory can represent today's advanced Hybrid recognition technology. But because of the limitation of the embedded platform in the aspects of resources and speed, the embedded implementation has still not been mature. While, because the algorithm of small vocabulary Hybrid commands recognition system is relatively simple, whose demand for resources is small, and the rate of high recognition and robustness is rather high, which can meet the requirements of most applications, thus it has become the main focus of the embedded application [3].

When the voice call begins, first of all, it should accept interface control information. This information can call the starting function of local voice and the starting function of the opposite terminal, judging whether it has opened coding thread or decoding thread, if it is not opened, then open it. The data after the coding thread is dealt with the data sending function, the data solved by decoding thread is dealt with the sound card to play.

When the voice call ends, first of all, it should accept interface control information. This information can call the termination function of local voice and the termination function of the opposite terminal, judging whether it has opened coding thread or decoding thread, if it is not opened, then open it $[4,5]$.

Hybrid recognition is a pattern recognition in essence, but its voice signal is more complicated, plus its content is quite rich, so Hybrid recognition is much complicated than the general pattern recognition, the English embedded interaction learning system mainly includes Hybrid signal 
pre-treatment, endpoint detection, feature parameter extraction, pattern matching, reference template library, and several other modules, the principle diagram of the system is shown in Figure 2.

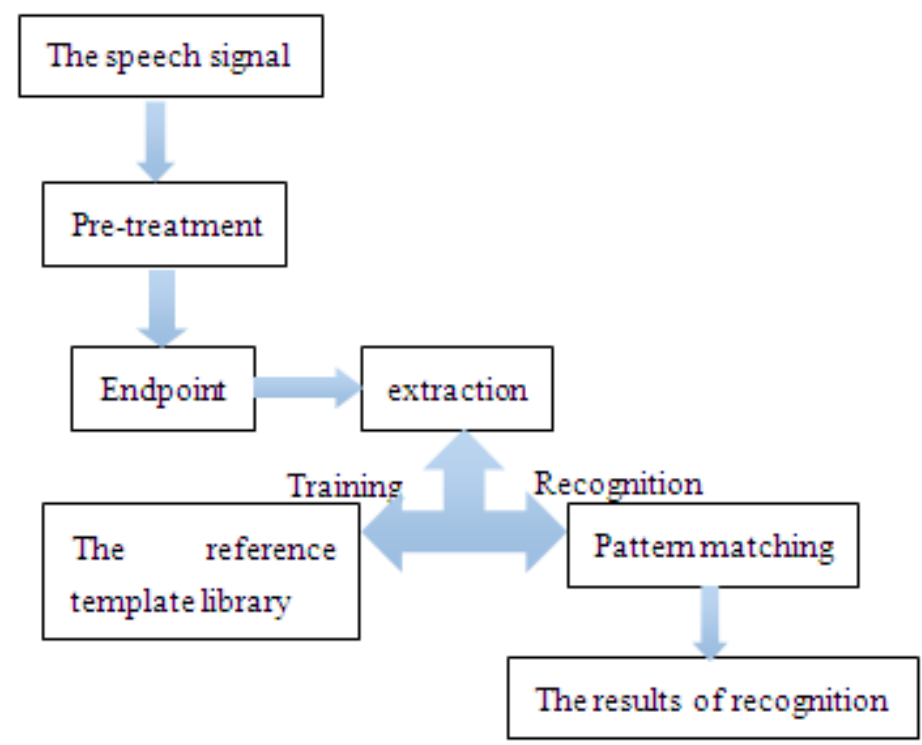

Fig.2 Block diagram of English embedded interaction learning system

The basic structure of English embedded interaction learning system includes: (1) pre-treatment includes the collection of Hybrid signal and the operation of the pre -emphasis, window adding and framing operation and so on; (2) the endpoint detection can separate the Hybrid signal effectively from the collected Hybrid signals; (3) feature parameter extraction refers to extract key characteristic parameters from the signals that can reflect the characteristics of the Hybrid signals; (4) the training phase refers to the acquired feature parameter vector after the Hybrid signal is input by the user, the pre-treatment of the Hybrid signal, endpoint detection and feature extraction, which takes the characteristic parameters of each Hybrid signal as the template and the formation of the reference template library. (5) the recognition stage refers to contrasting the similarity between the feature parameter vector of the unknown Hybrid signal and the reference template in the template library, taking the highest similarity model as the recognition results and then output.

Hybrid monitoring system of embedded PI transmission can realize the localization of the processing of Hybrid information, so as to improve the performance of the server. Each device can have access to the Internet with the service function, namely, for each Hybrid equipment, it can be regarded as an independent network terminal, thereby it can greatly improve the quality and scope of monitoring [6].

Embedded IP Hybrid equipment as well as CP are connected in the network, each Hybrid equipment of the whole system and CP can be regarded as the network device that has a unique PI address, thus each equipment can be recognized by the address of network PI. For each Hybrid equipment, the most basic function is to collect Hybrid, playback, compress the code and decode, act as network interface, etc. Each Hybrid equipment is equivalent to a Hybrid acquisition and monitoring equipment, they are working under the remote monitoring of $\mathrm{CP}$, which can complete the acquisition of data, compress the code and transmit the data. Remote CP can directly monitor the location of the Hybrid device and store the Hybrid information, when it is necessary, it can broadcast through the network in a single or multiple Hybrid broadcasting equipment.

\section{Conclusion}

In this paper, the implementation of the Hybrid engine based on Speex in Speaker independent English embedded interaction learning system is introduced in this paper, which has many advantages compared with the speaker dependent isolated word English embedded interaction learning system, thus it can become the main focus of researching the English embedded interaction learning system research as well as implementation. As for the embedded platform, researching and developing the 
front-end processing module of special Hybrid recognition can make it perform more complex Hybrid front-end signal processing algorithms. And it has performed well at this stage under the environment. It has showed the efficiency of Speex as the voice codec, as well as the processing ability in voice communication. English embedded interaction learning system has a broad prospect of market application.

\section{References}

[1] SAVOJIM H.1989, A robust algorithm for accurate endpointing of Hybrid. Hybrid Communications, vol.8, pp45-60.

[2] John M. Paulett, Curtis P. Langlotz. 2009, Improving language models for radiology Hybrid recognition. Journal of Biomedical Informatics, vol.42, pp53-58.

[3] Rabiner L, Juang B. 1993, Fundamentals of Hybrid Recognition. Englewood Cliff New Jersey Prentice-Hall.

[4] Pitton J, Atlas L, Loughlin P. 1994, Aplications of PossitiveTime-frequeney Distributions to Speeeh Proeessing. Speeeh and Audio Proeessing, vol.24, pp544-566.

[5] A.V.I Rosti, M.J. F Gales. 2004, Factor analysed hidden Markov models for Hybrid recognition. Computer Hybrid and Language, vol.18, pp181-200. 